\section{Complex PTSD in young people in care}

\section{Aishat Hamzat, Rachel M Hiller and Helen}

Minnis discuss what research tells us about the mechanisms underlying complex PTSD symptoms experienced by young people in care. They consider implications for treatment and broader support provided for these young people.

In March 2021 we joined CAMHS around the Campfire, the online journal club run by ACAMH and the Mental Elf, to discuss Rachel's recent paper on complex posttraumatic stress disorder (PTSD) in young people in care. ${ }^{1}$ Briefly, this paper presented results of a longitudinal study exploring the role of key cognitive predictors of both 'standard' PTSD and complex PTSD symptoms. The sample included 120 10-18 year-olds living in care in England. The study found that our current models of PTSD were applicable to young people in care. Maladaptive cognitions (e.g., "I can't trust anyone" or "the world isn't safe"), cognitive coping (e.g., avoiding thinking about what happened), and memory qualities (e.g., memories being muddled or confused) were all associated with PTSD symptoms and complex features. Maladaptive cognitions were particularly important. This suggests that targeting these mechanisms in intervention would be useful for young people with complex PTSD symptoms. In this article we reflect on some of the important points raised in the Campfire discussion about this research.

\section{Are PTSD and complex PTSD different?}

PTSD is a trauma-specific mental health difficulty. A young person who has experienced trauma (whether that might be called maltreatment, complex trauma, developmental trauma, or any other trauma exposure) is at risk of developing PTSD. According to the World Health Organisation's diagnostic classification system (ICD-11), PTSD includes three symptom clusters: re-experiencing the trauma,

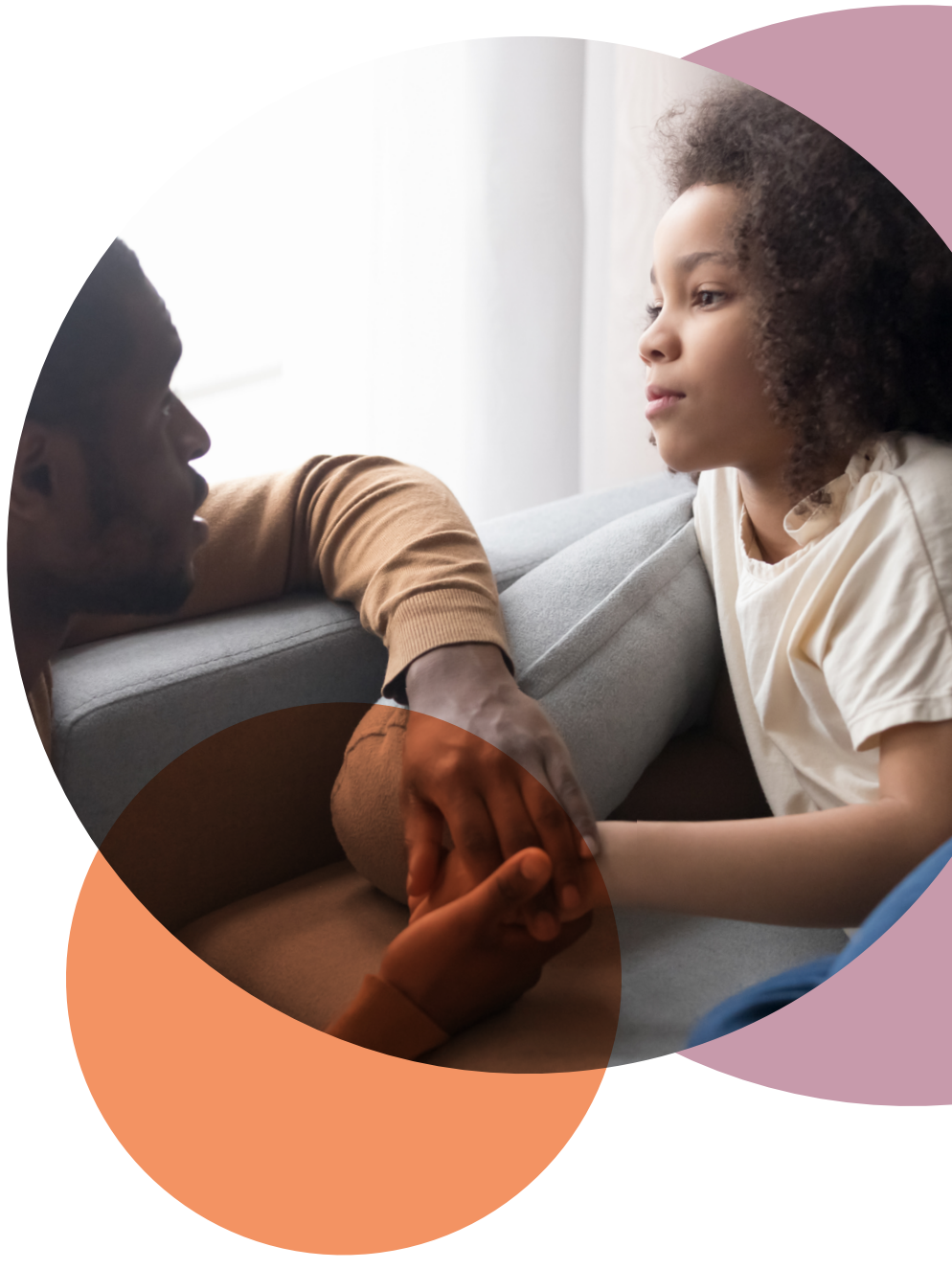

avoiding reminders of the trauma and hyper-arousal. Young people with PTSD commonly also have other mental health problems, such as depression or anxiety. We know that rates of PTSD are very high in care-experienced young people. Complex PTSD is a new diagnosis in the ICD-11, but has not been included in the American Psychiatric Association's diagnostic classification system (DSM-5). Crucially, to meet criteria for complex PTSD, the young person must have PTSD as well as additional complex features of difficulties with: relationships, emotion dysregulation and negative self-concept (e.g., "I'm not worth anything").

Rachel's work indicates that the same processes that drive PTSD, also drive complex PTSD. This suggests we may not need to develop completely new treatments for complex PTSD. We already have interventions that target these processes - called trauma-focused cognitive behaviour therapies (tf$\mathrm{CBT}$ ), the NICE-recommended first-line treatment 
for PTSD. ${ }^{2}$ There is growing research supporting this by showing that tf-CBT is an effective treatment for those with complex presentations. ${ }^{3}$ It's possible that young people with complex presentations may benefit from more sessions to allow a more in-depth focus on certain aspects of treatment.

\section{Getting young people in care the right treatment}

It's important that young people in care get the right treatment to address their needs. We know that even our best evidenced treatments do not work $100 \%$ of the time for $100 \%$ of young people, but they should be the starting point to giving young people the best chance at overcoming their mental health difficulty.

As with any treatment, it is also important that the young person receives appropriate psychoeducation, not just about PTSD, but also about the treatment. People with PTSD often want to avoid thinking about what they have been through, which means they might struggle to engage in earlier sessions or not turn up to some sessions. In the psychoeducation component of $\mathrm{tf}-\mathrm{CBT}$ it is important that avoidance is openly discussed. Similarly, it can be useful for practitioners to reflect on whether they may inadvertently encourage avoidance by delaying starting parts of treatment that are harder, such as memory work.

We also acknowledge the barriers that often exist in services that might mean practitioners find it difficult to deliver tf-CBT. It might be that they are not able to provide the required $12-20$ sessions, or that they do not have training and supervision available to them. But there also seems to be a pervasive belief that $\mathrm{tf}-\mathrm{CBT}$ is not appropriate for young people exposed to complex trauma, like abuse. This belief is not supported by the evidence or the most recent NICE review for PTSD, and is also challenged by mechanism research. Understanding how we can address service-level and individual-level barriers remains an important area of research, so practitioners and services can be encouraged and supported to deliver evidence-based treatments, such as tf-CBT.

\section{Listening to young people in care}

Another important issue is the need for researchers and practitioners to listen to, and genuinely hear, the views of young people in care. Young people in care are young people first - they are as different to each other as any group of young people. Some young people we have spoken to had gone for mental health support, only to feel let down by not receiving support that helped them. Some felt so let down that they decided never to engage with mental health services again. Aishat, a young person with care experience, spoke from her own experience about feeling patronised and let down by her initial contact with services. She felt that her initial experience seeking professional help was met with a lack of validation of the mental preparation it had taken her to even ask for help. Aishat felt that professionals often overrode her experience with their own assumptions. She talked about often feeling rushed and not being given the opportunity to talk about her experiences, and that she had sometimes felt that her memories were being invalidated or judged as biased or inaccurate. Aishat believes it is important to recognise the time it may take for a young person to truly scrutinise their trauma and accept its effects; bombarding them with information they're not ready to receive will only hinder rather than help the process. Because of the trauma experienced by young people in care, it seems we sometimes just accept that mental health difficulties are inevitable or unchangeable. We all agree that it is vital that we challenge this assumption, especially because young people might then internalise this message - e.g., "I'm too damaged to be helped", "no one can help me", "what I went through was so awful that not even adults want to hear me talk about it".

Related to this, we also discussed how important it is that professionals work alongside young people and consider their individual needs within their treatment plan. While young people may not always be able to label the psychological effects of their trauma, they are the expert in their own experience. We know that many young people in care have been let down by adults in their life (before care and in care) and might struggle with trust and relationships (this can be particularly true for those with PTSD). Building rapport is therefore very important.

\section{Future work}

There is much research to be done in the field of young people in care. Compared to research on the general population of young people, we are still far behind in what we know about the mental health needs of this groups. We all discussed the importance of moving this field forward with more large-scale projects, so that we can more strongly advocate for evidence-informed support for young people in care and care-leavers. 


\section{References:}

${ }^{1}$ Hiller RM et al (2021) A longitudinal study of cognitive predictors of (complex) posttraumatic stress in young people in out-ofhome care. J Child Psychol Psychiatry, 62, $48-57$.

${ }^{2}$ NICE (2018). Post-traumatic stress disorder: NICE guideline [NG116].

${ }^{3}$ Sachser et al (2017). Complex PTSD as proposed for ICD-11: validation of a new disorder in children and adolescents and their response to trauma-focused cognitive behavioral therapy. J Child Psychol Psychiatry, 58, 160-168.
:

$\square$ Campfire: Webinar discussion of this topic

滋 Scotland's Independent Care

1 Review: The promise

湔 Child Trauma Recovery:

$\square$ Evidence- based information for parents and carers

To visit any of the links related to this article, click here to go to the ACAMH website.

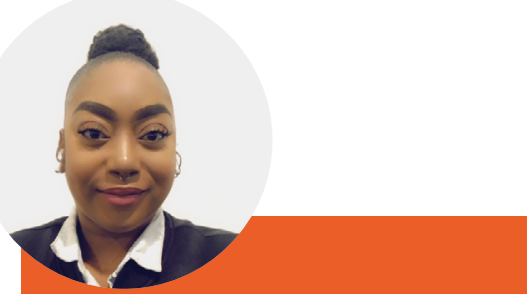

Aishat Hamzat is a care leaver, student and volunteer campaigns advisor for the National Youth Advocacy Service.

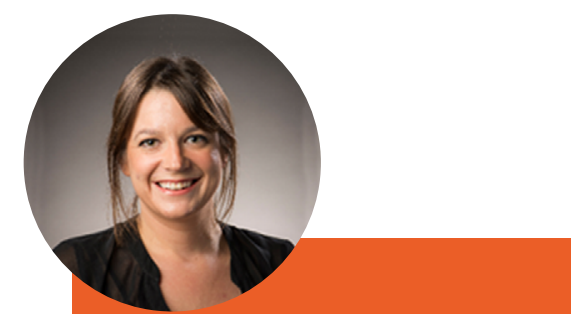

Dr Rachel M Hiller is a Reader in Child and Adolescent Clinical Psychology in the Department of Psychology, University of Bath. Her research examines cognitivebehavioural processes that link the experience of child trauma or maltreatment to psychological outcomes, with a focus on (complex) posttraumatic stress disorder. Her work is also particularly focused on improving the mental health outcomes of young people in the child welfare system, including testing new cross-sector interventions for PTSD and improving access to existing evidence-based treatments.

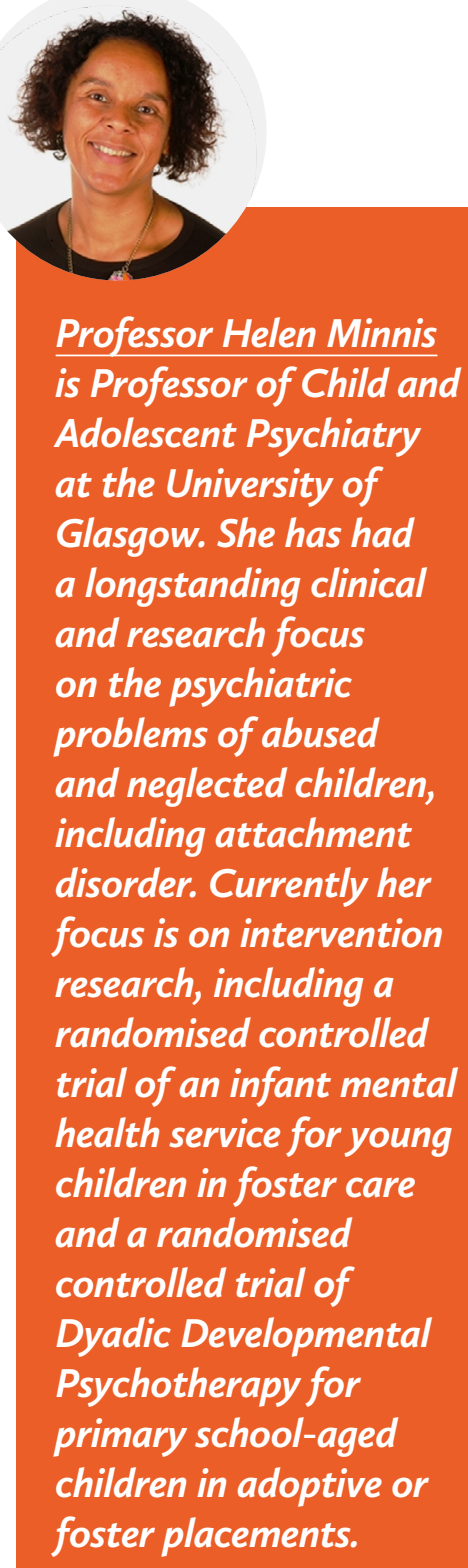

